\title{
Neutron Bang Time Detector Based on a Light Pipe
}

V. Yu Glebov, M. Moran, C. Stoeckl, T. C. Sangster, M. Cruz

May 12, 2008

HTPD-17 Seventeenth High-Temperature Plasma Diagnostics Conference Albuquerque, NM, United States May 11, 2008 through May 15, 2008 
This document was prepared as an account of work sponsored by an agency of the United States government. Neither the United States government nor Lawrence Livermore National Security, LLC, nor any of their employees makes any warranty, expressed or implied, or assumes any legal liability or responsibility for the accuracy, completeness, or usefulness of any information, apparatus, product, or process disclosed, or represents that its use would not infringe privately owned rights. Reference herein to any specific commercial product, process, or service by trade name, trademark, manufacturer, or otherwise does not necessarily constitute or imply its endorsement, recommendation, or favoring by the United States government or Lawrence Livermore National Security, LLC. The views and opinions of authors expressed herein do not necessarily state or reflect those of the United States government or Lawrence Livermore National Security, LLC, and shall not be used for advertising or product endorsement purposes. 


\title{
Neutron Bang Time Detector Based on a Light Pipe
}

\author{
V. Yu. Glebov ${ }^{1}$, M. Moran ${ }^{2}$, C. Stoeckl ${ }^{1}$, T. C. Sangster ${ }^{1}$, M. Cruz ${ }^{1}$ \\ ${ }^{1}$ Laboratory for Laser Energetics, University of Rochester, 250 East River Road, \\ Rochester, NY 14623 \\ ${ }^{2}$ Lawrence Livermore National Laboratory, 7000 East Avenue, Livermore, CA 94550 \\ (585) 275-7454,vgle@lle.rochester.edu
}

\begin{abstract}
A neutron bang time detector consisting of a scintillator, light pipe, photomultiplier tube (PMT), and high-bandwidth oscilloscope has been implemented on the 60-beam, 30-kJ OMEGA Laser Facility at the University of Rochester's Laboratory for Laser Energetics. Light from the scintillator, located $23 \mathrm{~cm}$ from the target, is transmitted outside the target bay through a 9.6-m-long, 2-in.-diam polished stainless steel pipe to the PMT. The PMT signal is recorded by two channels of a 6-GHz, 10-GS/s Tektronix 6604 oscilloscope. The OMEGA optical fiducial pulse train is recorded on the third oscilloscope channel using a fast photodiode to provide the timing reference to the laser. The bang-time detector is absolutely calibrated in time and is able to measure bang time for neutron yields above $1 \times 10^{9}$ with accuracy of better than 25 ps.
\end{abstract}

Lawrence Livermore National Laboratory is operated by Lawrence Livermore National Security, LLC, for the U.S. Department of

Energy, National Nuclear Security Administration under Contract DE-AC52-07NA27344. 


\section{Introduction}

In the Inertial Confinement Fusion (ICF) [1] experiments a target filled with a fuel is heated either by direct laser illumination or by soft x-ray radiation in a laser heated hohlraum. In the resulting implosion thermonuclear fusion occurs and charged particles, photons, and neutrons are being released. The time interval from the beginning of the laser pulse irradiating the target to the peak of neutron emission - the neutron bang time is very sensitive to the details of the energy absorption and the hydrodynamic response of the target. Therefore the bang time is an important parameter of the ICF implosion providing information on the target performance which can be compared with numerical models. The 60-beam, 30-kJ OMEGA Laser Facility [2] at the University of Rochester's Laboratory for Laser Energetics has several diagnostics [3-6] to measure neutron bang time. These include a fast ( $<25$ ps) streak camera-based neutron temporal diagnostic (NTD) [3] and a neutron bang time (NBT) [5] detector using scintillator coupled to photomultiplier tube (PMT) and recording signal on oscilloscope. This paper describes a new light pipe neutron bang time detector (LPNBT), which consists of a fast scintillator, light pipe [7], PMT, and high-bandwidth oscilloscope. The LPNBT utilizes the light pipe platform created on OMEGA in order to develop a gamma bang time [8] detector for the National Ignition Facility (NIF) [9]. The performance of the light pipe based gamma bang time detector will be described in a separate paper.

\section{LPNBT Design and Setup}

The LPNBT diagnostic design is shown in Fig. 1. A $22 \mathrm{~mm}$ diameter, 1mm thick ultrafast BC-422 plastic scintillator [10] located at $23 \mathrm{~cm}$ from the OMEGA target 
chamber center. The BC-422 scintillator has less than 20 ps rise time [11] and $1.4 \mathrm{~ns}$ decay time. A 3-mm-thick tungsten filter is located in front of scintillator as $\mathrm{x}$ ray shielding. Light from the scintillator propagates first through a 1-in. diameter, $17 \mathrm{~cm}$ long light pipe following by 2-in. diameter, 9.43-m long light pipe. The light pipe [7] is a multiple-section stainless steel tube with polished interior. One turning mirror is used to combine two straight sections of the light pipe and redirect light to the room below Target Bay called La Cave on OMEGA. The light pipe with the scintillator is placed in a reentrant tube inserted into OMEGA target chamber. To provide $\mathrm{x}$ ray timing calibration the front part of reentrant tube shown on Fig. 1 is made from aluminum with a wall thickness $1 \mathrm{~mm}$. The back side of the reentrant tube is made from stainless steel. Since the light pipe and scintillator assembly is COMPOSED of multiple sections, the scintillator or front shielding can be changed quickly. At the end of light pipe a spherical lens with $102 \mathrm{~mm}$ focal length is used to focus the scintillator light onto a R3809U-52 Hamamatsu microchannel plate PMT. The PMT has a $11 \mathrm{~mm}$ photocathode, 136 ps rise time and was operated at $10^{5}$ gain. A 10 -m-long LMR-400 coaxial cable connects the PMT to a 6-GHz, 10-GS/s, Tektronix TDS 6604 oscilloscope. A resistive splitter divides the PMT signal between two oscilloscope channels with different sensitivity settings to increase the dynamic range of the recording system. The OMEGA optical fiducial pulse train is recorded on a third channel, using a fast photodiode to provide a time reference to the laser. The fiducial analysis and fitting procedure of the fiducial pulse train are described in Ref. 5.

Both LPNBT and NBT [5] use fast scintillator, PMT, and high bandwidth oscilloscope. The main difference between LPNBT and NBT is to use of light pipe to 
separate PMT and scintillator. This allows placing scintillator close to a target, putting no restriction on the size of PMT, and drastically decreasing neutron background and electromagnetic pulse noise in the PMT. The PMT location outside of the Target Bay makes it more practical to put neutral density filters as needed in front of the PMT eliminating possible PMT saturation. As a result useful dynamic range of the LPNBT was increased in comparison with NBT.

\section{LPNBT Performance}

The LPNBT was tested on OMEGA with both DT and $\mathrm{D}_{2}$ implosions. Figure 2a shows the oscilloscope trace of the LPNBT for a $\mathrm{D}_{2}$ shot yielding $2.75 \times 10^{11}$ neutrons. The signal from the PMT was attenuated by a high bandwidth $20 \mathrm{~dB}$ attenuator in order not to saturate oscilloscope. The signal attenuation and splitting into two oscilloscope channels was taken into account during analysis. The measured signals were fit by a convolution of a Gaussian and an exponential decay, as described in Ref. 4. The falling edge of the pulse was fitted only to $50 \%$ of the peak amplitude eliminating interference from re-scattered neutron after the main primary neutron peak. The exponential decay parameter in LPNBT fit was chosen to be 1.5 ns. It represents mainly the BC-422 scintillator decay (1.4 ns) but also includes a small contribution from PMT, cable, and oscilloscope response time. The exponential decay parameter for the LPNBT was fixed for all type of shots (hard x-ray, $\mathrm{D}_{2}$, and DT). The Gaussian parameters (width, amplitude, and position) are free parameters of the fit for every shot to account for different yields, bang times, ion temperatures, and trigger shifts. The neutron pulse arrival time is defined to be the center of the Gaussian portion of the fit. Figure $2 \mathrm{~b}$ shows the 
oscilloscope trace of the LPNBT for a DT shot yielding $1.1 \times 10^{12}$ neutrons. In case of DT implosions yields are about 100 times higher than in $\mathrm{D}_{2}$ implosions and can easily saturate PMT. Therefore, in DT implosion neutral density filters were used to attenuate light in front of PMT and expand the dynamic range of the LPNBT diagnostic. Figure 3 shows the signal amplitude of the LPNBT channel as a function of $\mathrm{D}_{2}$ and DT neutron yield. Since the neutron yield is proportional to the integral of the signal and not the amplitude $\mathrm{D}_{2}$ and DT implosions may show different amplitudes for the same yield because of different ion temperature. The low neutron yield is limited by neutron statistics and although LPNBT recorded signals with yields from $5 \times 10^{8}$, the good pulse fit and timing accuracy starts from $1 \times 10^{9}$ yields. The amplitude in Fig. 3a is real amplitude from PMT and effective amplitude in Fig. $3 \mathrm{~b}$ is amplitude with neutral density filters attenuation taken into account. The LPNBT recorded much more data in $\mathrm{D}_{2}$ implosions than in DT implosions. This reflects OMEGA schedule and the fact that in most high yield DT shots the light pipe was used for gamma bang time development. The LPNBT was tested with yields up to $3 \times 10^{13}$. With appropriate neutral density filters there is no limit for LPNBT in signal saturation. While NTD is limited by neutron induced background in the steak camera the PMT and oscilloscope in LPNBT location are not very sensitive to neutron background. It is known from gamma bang time experiments that the light pipe PMT and oscilloscope operate successfully in the neutron background from the yields of up to $1 \times 10^{14}$. Therefore, the LPNBT diagnostic can measure the neutron bang time in implosions over the yield range from $1 \times 10^{9}$ to at least $1 \times 10^{14}$ or maybe even higher. 
The timing accuracy of the LPNBT was studied by comparison of the neutron bang time measured on the same shots by LPNBT and NTD. Figure 4a shows the cross calibration of the LPNBT and the NTD performed during two days of D2 implosions with a total of 14 shots, two shell thicknesses and yields varying from $2.3 \times 10^{10}$ to $4.9 \times$ 1010. A good correlation between the NTD and the LPNBT is observed with an rms difference of 25 ps. This is less than the 28 ps rms expected for the difference between two independent measurements each with a time precision of 20 ps. Figure $4 \mathrm{~b}$ a similar plot for one day of DT implosions with different shell thicknesses and yields varied from $3.8 \times 10^{11}$ to $7.6 \times 10^{11}$. The rms of the time difference between the two diagnostics is 10 ps, but number of shots is very limited.

There are two options for the calibration of the LPNBT bang time relative to the OMEGA laser pulse; first by cross calibration against NTD as shown on Fig. 4, and second by the LPNBT independent calibration using hard $\mathrm{x}$-ray produced by a gold coated target irradiated with a short 100 ps laser pulse at best focus. To obtain a measurable signal for this calibration the tungsten shielding in front of the LPNBT scintillator was replaced with $1 \mathrm{~mm}$ thick aluminum filter. Figure 5 shows an x-rayinduced signal recorded by the LPNBT, a curve fit to this signal using the expression described in the previous section, and the shape of the 100-ps laser pulse as recorded by the OMEGA UV streak camera system. The effect of the limited bandwidth and the scintillator decay are clearly seen in the recorded signal, but the position of the signal peak can be determined accurately using the fitting procedure. The x-ray calibration can be easily carried over to the neutron measurements using the propagation delay difference from the target to the scintillator between $\mathrm{x}$ rays and neutrons. A measurement 
uncertainty in the scintillator distance on the order of $0.5 \mathrm{~mm}$ corresponds to a calibration error of $25 \mathrm{ps}$ for $\mathrm{D}_{2}$ and $10 \mathrm{ps}$ for DT neutrons.

\section{ACKNOWLEDGMENT}

This work was supported by the U.S. Department of Energy Office of Inertial Confinement Fusion under Cooperative Agreement No. DE-FC52-08NA28302, the University of Rochester, and the New York State Energy Research and Development Authority. The support of DOE does not constitute an endorsement by DOE of the views expressed in this article.

\section{REFERENCES}

1. J. D. Lindl, Inertial Confinement Fusion: The Quest for Ignition and Energy Gain Using Indirect Drive (Springer-Verlag, New York, 1998).

2. T. R. Boehly et al., Opt. Commun. 133, 495 (1997).

3. R. A. Lerche et al, Rev. Sci. Instrum. 66, 933 (1995).

4. C. Stoeckl et al., Rev. Sci. Instrum. 74, 1713 (2003).

5. C. Stoeckl et al., Rev. Sci. Instrum. 73, 3796 (2002).

6. V. Yu. Glebov et al., Rev. Sci. Instrum. 77, 10E712 (2006).

7. M. Moran et al., Rev. Sci. Instrum. 77, 10E718 (2006).

8. V. Yu. Glebov et al., Rev. Sci. Instrum. 77, 10E715 (2006).

9. E.I. Moses, Fusion Sci. Technol. 44, 11 (2003).

10. www.bicron.com

11. R.A. Lerche et al., SPIE, 1993, vol. 2002, p. 153. 


\section{FIGURE CAPTIONS}

FIG. 1. (E16699) Design of the LPNBT diagnostic.

FIG. 2. (E16701) The LPNBT signals:

a) for shot 46165 with a $D_{2}$ neutron yield $2.75 \times 10^{11}$,

b) for shot 47575 with a DT neutron yield $1.1 \times 10^{12}$.

FIG. 3. (E16702) Signal amplitude of the LPNBT versus neutron yield:

a) for $\mathrm{D}_{2}$ implosions,

b) for DT implosions.

FIG. 4. (E16703) Timing cross-calibration between the LPNBT and the NTD:

a) for D2 implosions,

b) for DT implosions.

A line of equal bang time for both instruments is shown in the graph for comparison.

FIG. 5. (NBTB Detail plot) LPNBT signal and corresponding fit for a hard x-ray emission produced in shot 50096 by a 100-ps laser pulse.

\footnotetext{
Disclaimer

This document was prepared as an account of work sponsored by an agency of the United States government. Neither the United States government nor Lawrence Livermore National Security, LLC, nor any of their employees makes any warranty, expressed or implied, or assumes any legal liability or responsibility for the accuracy, completeness, or usefulness of any information, apparatus, product, or process disclosed, or represents that its use would not infringe privately owned rights. Reference herein to any specific commercial product, process, or service by trade name, trademark, manufacturer, or otherwise does not necessarily constitute or imply its endorsement, recommendation, or favoring by the United States government or Lawrence Livermore National Security, LLC. The views and opinions of authors expressed herein do not necessarily state or reflect those of the United States government or Lawrence Livermore National Security, LLC, and shall not be used for advertising or product endorsement purposes.
} 


\section{The LPNBT consists of a fast scintillator, light pipe ${ }^{*}$, PMT, and a fast digital oscilloscope}

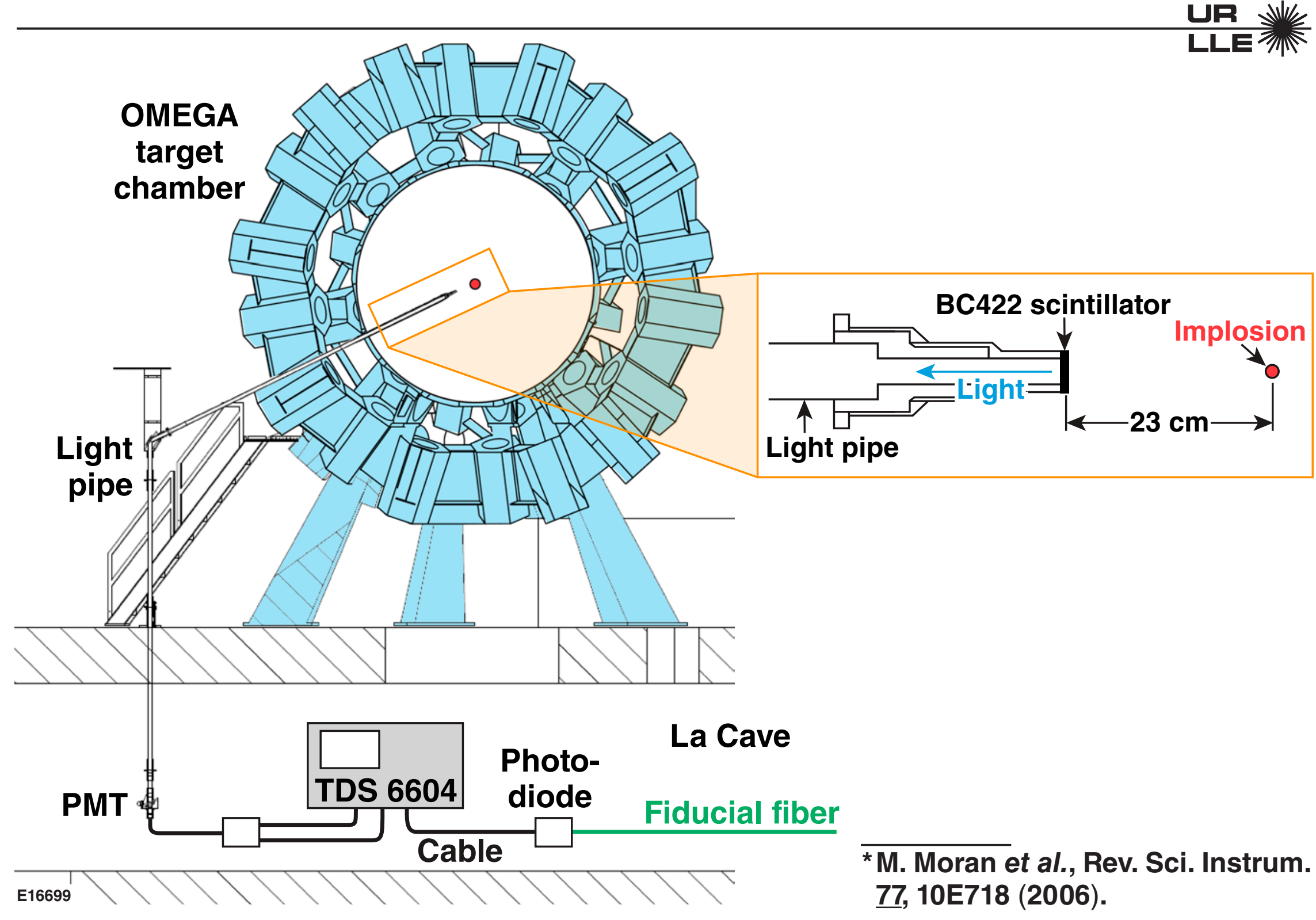


The LPNBT signals $m(t)$ are fitted by a convolution of a Gaussian and an exponential decay function

LLE 潘

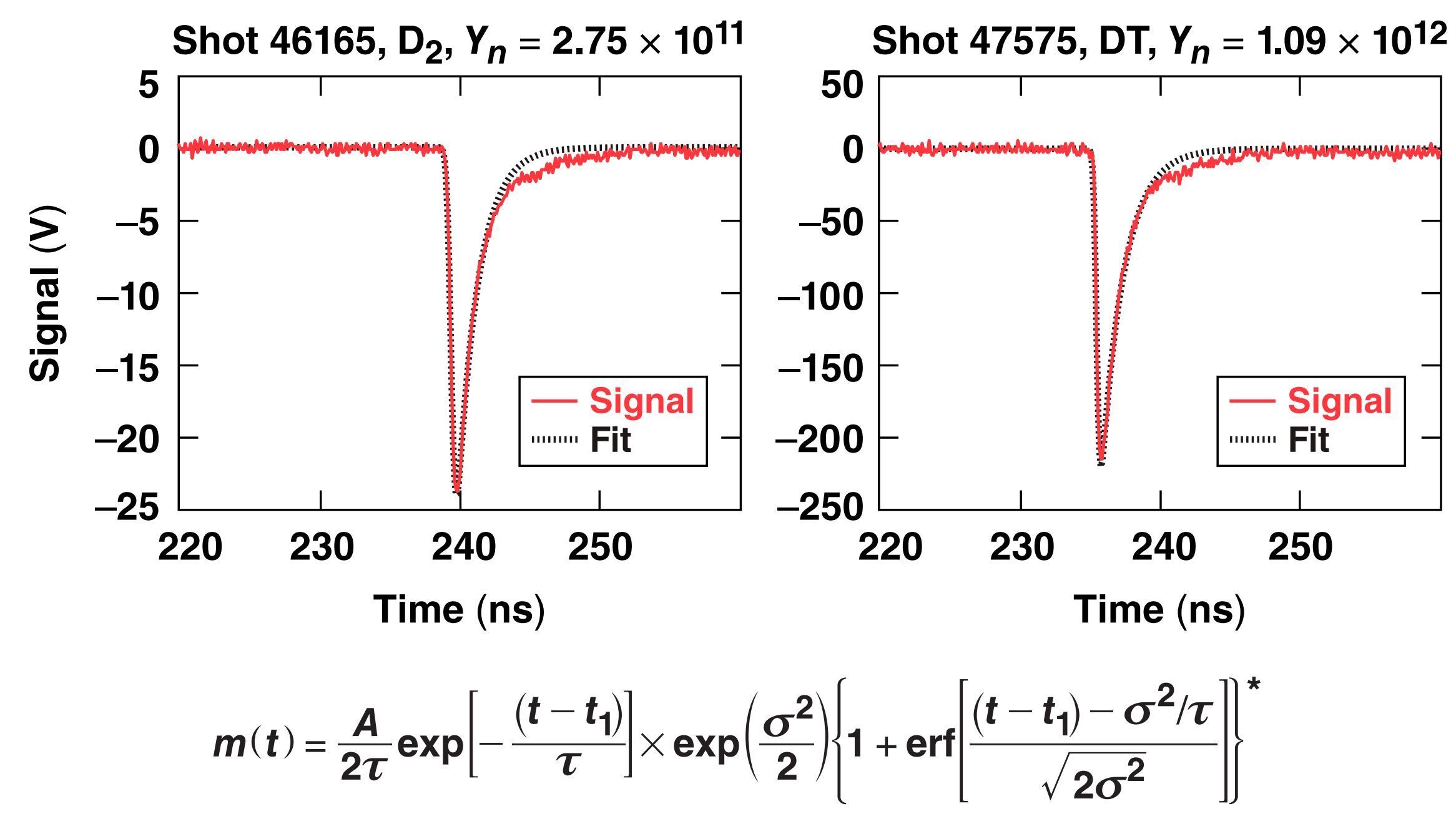


The LPNBT can measure the neutron bang time in direct-drive implosions from $1 \times 10^{9}$ to $1 \times 10^{14}$
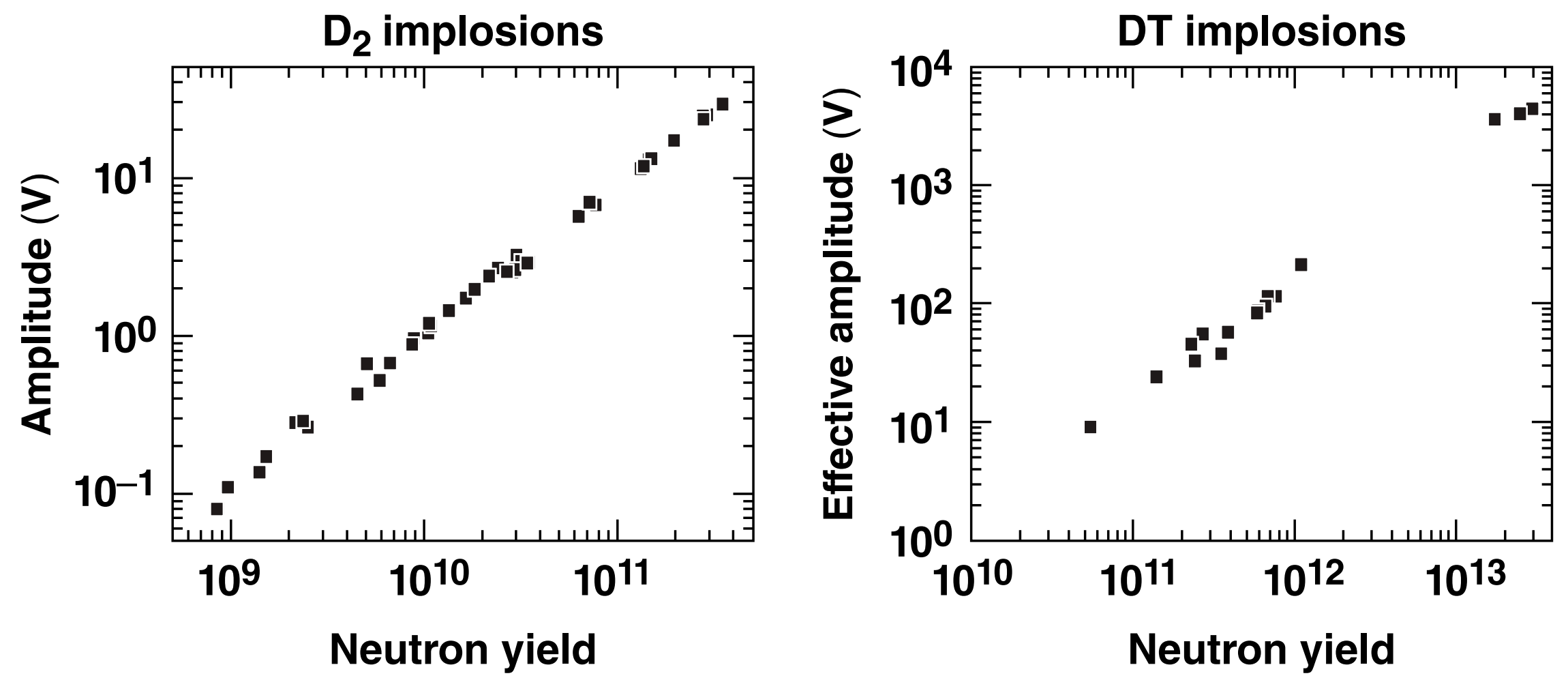

Neutral density filters in front of the PMT can be used to increase the dynamic range for both $D_{2}$ and DT implosions. 
The LPNBT timing accuracy was compared with the streak-camera-based neutron temporal diagnostic (NTD)
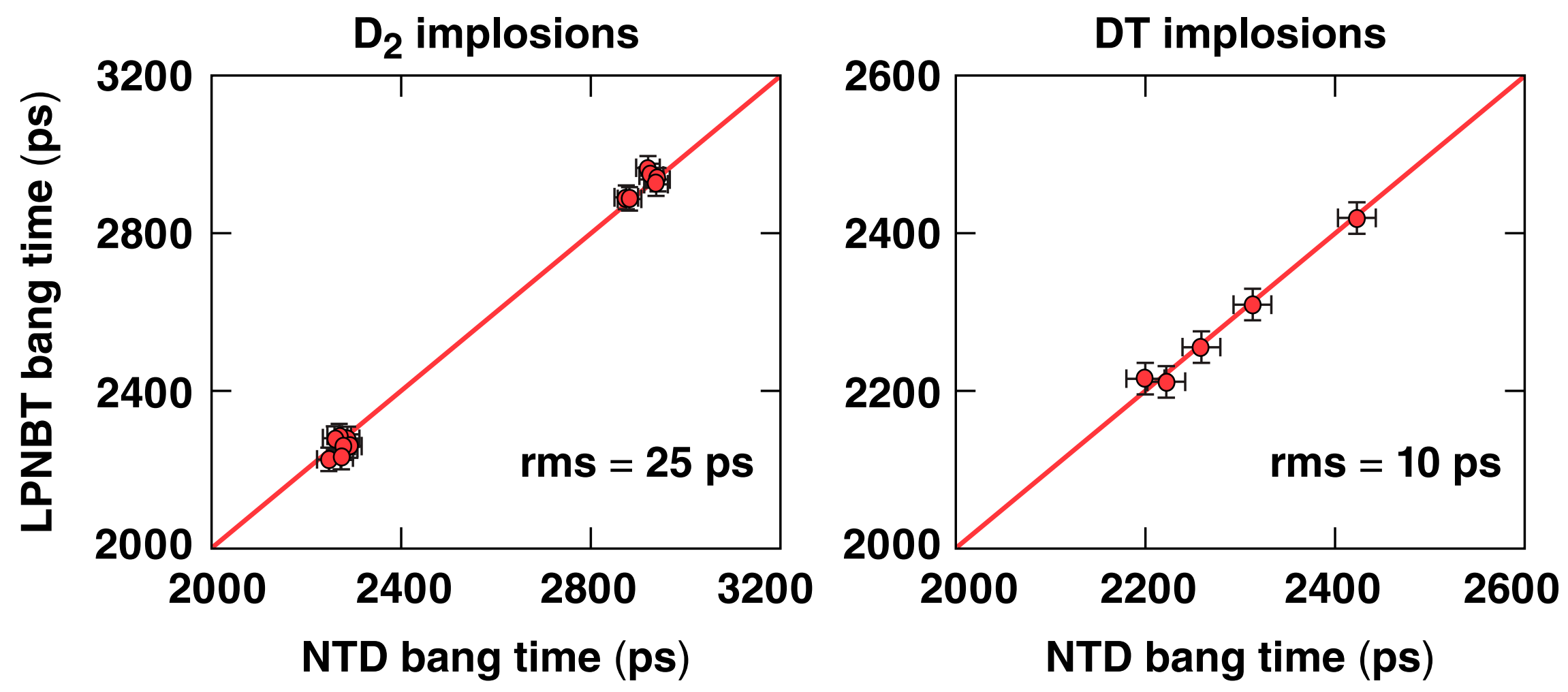

- Bang-time data

- Function $y=x$ 


\section{NBT ANALYSIS}

Bang Time Front: 20035
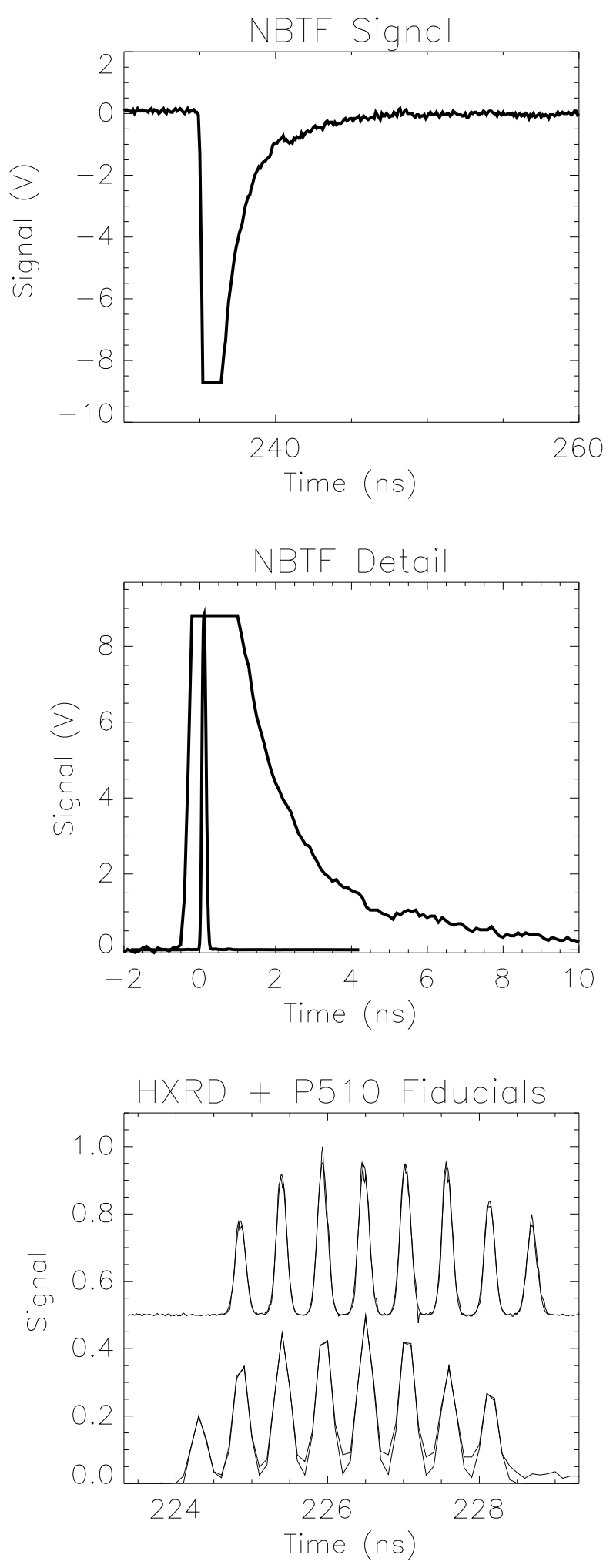

SHOT: 50096

Bang Time Back: -191
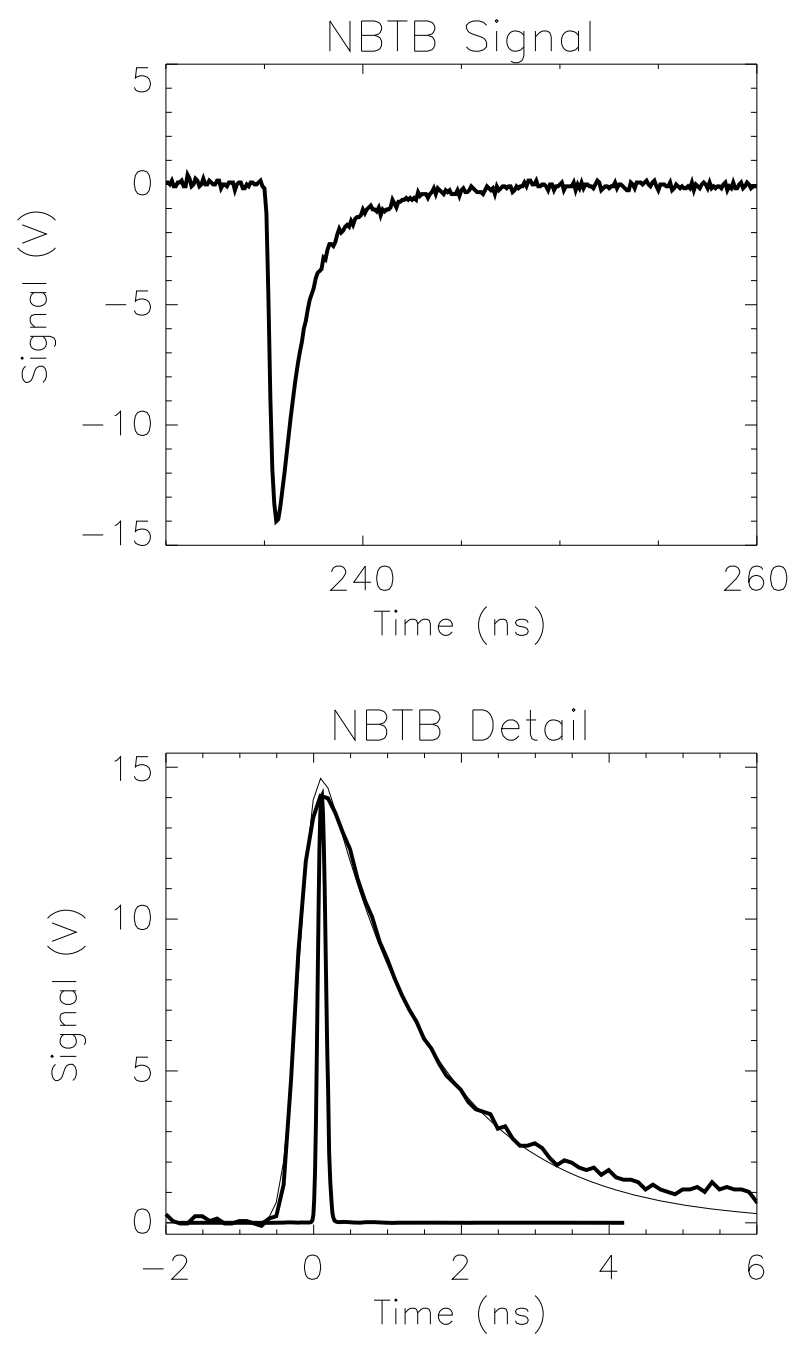

NBT FiduRef: 224.31

P510 FiduRef: 0.89 Cluster: 4

NBTF Min: $\quad-8.72$

NBTF Chi:

1000.00

NBTB Min:

$-14.00$

NBTB Chi:

1.19

NBTF NeutRef: -10.09

NBTB NeutRef: -4.68

NBTF Calib : 10.20

NBTB Calib : 10.30 